\title{
Septic ischial bursitis in patients with spinal cord injury
}

\author{
S Rubayi MD FICS, ${ }^{1} \mathrm{~J}_{\mathrm{Z}}$ Montgomerie FRACP ${ }^{2}$ \\ ${ }^{1}$ Pressure Ulcer Management Service, Department of Surgery, Rancho Los Amigos \\ Medical Center, Downey, California 90242, USA; ${ }^{2}$ University of Southern California \\ School of Medicine, Los Angeles, California 90033 and Infectious Disease Division, \\ Department of Medicine, Rancho Los Amigos Medical Center, Downey, California \\ 90242 USA.
}

Septic ischial bursitis is described in 4 patients with spinal cord injury. In these patients a pre-existing ischial bursitis probably became secondarily infected. Because these patients lack sensation, diagnosis may be difficult. The disease process in one patient with a prolonged fever was only recognized after a leucocyte scan detected an abscess extending to the thigh. At surgery it was found that the infection extended from the ischial bursa to the upper lateral thigh. Infection in these patients was due to beta hemolytic streptococcus, ${ }^{1} S$. aureus, ${ }^{1}$ and $S$. epidermidis. ${ }^{2}$ The patients all responded well to local drainage and excision of the bursa.

Key words: septic ischial bursitis; spinal cord injury.

\section{Introduction}

Ischial bursitis (also known as Weaver's or Tailor's bottom), a disease that has been associated with trauma from sitting for long periods of time, is usually associated with perianal pain. ${ }^{1}$ Ischial bursitis is not uncommon in patients with spinal cord injury ${ }^{2}$ where it may be a significant component of ischial pressure ulcers. ${ }^{3,4}$ These ulcers may not heal without excision of the bursa, and the bursa may recur after musculocutaneous flap surgery.

In those patients without pressure ulcers the problem may not be recognized because patients with spinal cord injury lack sensation. We recently treated a patient with spinal cord injury who appeared to have an ischial bursitis that became infected and presented with a fever of unknown origin. We describe this patient and 3 additional patients with spinal cord injury and infected ischial bursitis.

\section{Case 1}

This 29 year old female T4/T5 paraplegic was injured in 1975, and had competed in wheelchair racing for many years. Apart from a recent pharyngitis she had been well until she developed a fever $\left(102-103^{\circ} \mathrm{F}\right)$. On admission to the hospital her physical examination was consistent with T4/T5 paraplegia with no other abnormalities and investigations at that time showed a leucocytosis (WBC $\left.21,000 \times 10^{9} / \mathrm{L}\right)$. A urine specimen cultured $E$. coli, but this was not felt to be the source of the fever. A leukocyte scan showed increased activity in the medial aspect of the right hip. Repeat physical examination of the area did not show swelling or erythema. Ultrasound of the area showed an abscess. Aspiration and culture of the abscess was positive for beta hemolytic streptococcus. A large abscess extending from the lateral mid thigh to the ischial tuberosity was drained and the wound left open, and she had systemic antibiotics. Drainage from the wound continued for 2 months and at further surgery a very large ischial bursa extending under the gluteal muscle and inferiorly over the hamstring muscle and laterally to the greater trochanter area was found, and was excised. The wound was closed without any flap procedure and healed without complication.

\section{Comment}

We believe that the ischial bursitis developed as a result of the wheelchair racing 
and was not detected because of lack of sensation and lack of local swelling or redness. The bursa may have become infected with the beta hemolytic streptococcus at the time of the pharyngitis, a few days before the patient developed a high fever. Even after the infection was detected in the thigh by leucocyte scan, the extensive infected bursa could not be detected on physical examination. Only at the time of surgery was it recognized that the infection had originated in the ischial bursa. The drainage persisted for over 2 months, and discontinued only after the bursa was excised.

\section{Case 2}

This 42 year old male, a T6 traumatic paraplegic, developed a swelling over the left ischial region which gradually increased in size over 2 years. There was no history of fever and chills or previous skin breakdown. Clinical examination revealed a soft fluctuating mass over the ischial region, about $6 \mathrm{x}$ $8 \mathrm{~cm}$ in size (Fig 1). X-rays of the pelvis and hip joint were within normal limits. A clinical diagnosis of ischial bursitis was made and the bursa was surgically exposed through the lower gluteal muscle approach. Complete excision of the bursa was followed by a rotation flap using the lower portion of the gluteus maximus muscle. Cultures from the bursal cavity grew $S$. epidermidis and histology of the bursa showed chronic synovitis. Bone biopsy from the ischium showed normal bone histology. The wound healed completely without further problems.

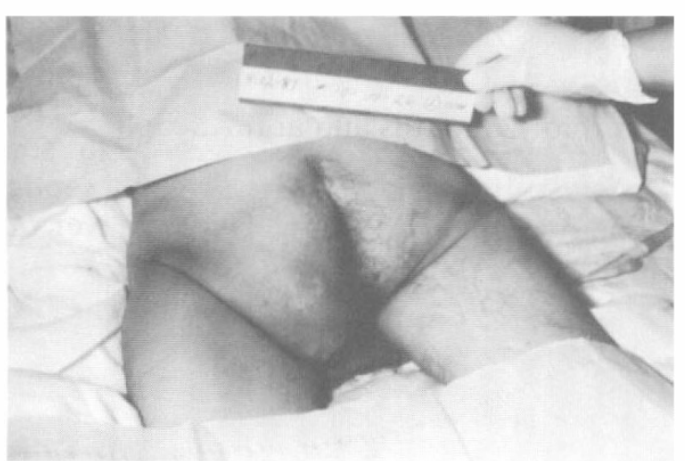

Figure 1 Large mass over left ischial tuberosity of patient (Case 2).

\section{Comment}

This patient developed ischial bursitis associated with obvious swelling over the ischial tuberosity over 2 years. Although $S$. epidermidis was cultured at the time of surgery, there was no other evidence of infection.

\section{Case 3}

This 28 year old female, with T12 complete traumatic paraplegia, developed a swelling over the left buttock area a few weeks following a fall and injury to her buttock. The patient denied fever and chills. On physical examination there was fluctuating swelling over the left ischial region.

Fluid aspirated from the swelling was turbid and red in color and contaned $3.7 \times 1^{-10} / \mathrm{L} \mathrm{RBC}$ and $0.9 \times 10^{9} / \mathrm{L}$ WBC. $S$. epidermidis was cultured from the aspirate. The peripheral WBC count was $6.3 \times 10^{9} / \mathrm{L}$ with normal differential. The bursal swelling was surgically excised and this defect was closed with a gluteus maximus muscle rotation flap. The patient was treated with cefazolin for 7 days.

Histology of the bursal tissue showed synovitis with mild chronic and acute inflammation. Bone histology showed no evidence of osteomyelitis. The wound healed completely without complication.

\section{Comment}

Injury with or without hematoma may also precede septic bursitis at other sites such as olecranon and prepatellar bursae.

\section{Case 4}

This patient was an 18 year old male with low level myelomeningocele and a long standing history of right ischial ulcer, treated with a gluteus maximus flap that was revised one year later. Three weeks after discharge from the hospital he was readmitted with a history of fever of $102^{\circ} \mathrm{F}$ and swelling in the right ischial region of one day duration. On examination, a fluctuating swelling over the right ischial region in the previous flap scar was seen. Pus that was aspirated cultured $S$. aureus. The peripheral WBC was $10.6 \times 10^{9} / \mathrm{L}$. The swelling was 
drained surgically and antibiotics were given for 14 days. The bursa was then excised completely and the wound closed by advancement of the old flap. The ischial bone biopsy taken for histology showed no evidence of osteomyelitis, and cultures were negative. The wound healed completely without complication.

\section{Comment}

This is the more common presentation of ischial bursitis after an ischial pressure ulcer. Although he had previous surgeries with removal of the bursal sac, it had redeveloped and became infected after myocutaneous flap surgery.

\section{Discussion}

Shea ${ }^{2}$ described the pathogenesis of ischial bursitis in patients with spinal cord injury, and called it the closed pressure ulcer. Ischial bursitis is seen mainly in the thin patient with prominent ischial tuberosity or pelvic deformity. Sitting for long periods of time with excessive shearing forces over the ischial area predisposes to bursal development. In patients with normal sensation the main clinical features are buttock pain that may radiate down the leg, ${ }^{1}$ pain that is aggravated by sitting, and tenderness and swelling over the ischial tuberosity. The tender bursa may be felt in the lateral wall on rectal examination. The bursa can reach an enormous size; an enlarged bursa weighing 2 lbs was removed surgically in $1932.5^{\circ}$

Ischial bursitis may be a diagnostic problem in patients with spinal cord injury because they lack sensation. Comarr ${ }^{3}$ and Constantian ${ }^{4}$ described the existence of atypical ischial bursa with ischial ulcer. In the 4 patients that we have described, the diagnosis was difficult even after the bursa became infected.

There are few descriptions of infected ischial bursitis. Infection of the ischial bursa has been described in a patient with systemic lupus erythematosus. ${ }^{6}$ A large ischial bursa secondarily infected with tuberculosis that involved the ischium was described by Chafetz et al. ${ }^{7}$

We have described 4 patients with infected ischial bursitis. The 2 patients with positive cultures for $S$. epidermidis had limited evidence of infection other than inflammatory changes on the histopathology.

The bacteria isolated from the infected bursitis in our experience (Streptococcus hemolyticus, Staphylococcus epidermidis and Staphylococcus aureus) were similar to microorganisms isolated from septic bursitis elsewhere in the body ${ }^{8-10}$ and were different from the bacteria isolated from patients with infected pressure ulcers.

Managing ischial bursitis depends on the clinical presentation. If the patient presents with gluteal swelling, diagnostic aspiration of the bursa should be done. Surgery comprises exploration of the bursa. Surgical drainage of the bursa should be done in the gluteal crease to help further closure with myocutaneous flap. The patient should be treated with appropriate antibiotics. Later, when the infection subsides completely, excision of the bursa and closure with gluteus maximus myocutaneous flap can be done. If the ischial tuberosity is found to be involved, a bone biopsy may be obtained to exclude osteomyelitis. Osteomyelitis of the ischial tuberosity, however, can usually be managed by local debridement, and does not require long courses of antibiotics. ${ }^{11}$

Many disorders including abdominal diseases $^{12}$ and spinal osteomyelitis ${ }^{13}$ may be difficult to diagnose in patients with spinal cord injury because they lack sensation. The absence of pain is also a problem for patients with spinal cord injury and septic ischial bursitis who may develop a fever of unknown origin.

\section{References}

1 Swartout R, Compere ED (1974) Ischiogluteal bursitis. The pain in the arse. JAMA 225: 551.

2 Shea JD (1975) Pressure sores. Clin Orthop 112: 89.

3 Comarr AE (1950) Ischial decubitus ulcer with atypical features. J Int Coll Surg 13: 232.

4 Constantian MB (1980) Pressure Ulcers, Principles and Techniques of Management. Little-Brown, Boston.

5 Frazer IF (1932) A very large bursa. Lancet I: 290-291. 
6 Lambie P, Kaufman R, Beardmore T (1989) Septic ischial bursitis in systemic lupus erythematosus presenting as a perirectal mass. J. Rheumatol 16: 1497-1498.

7 Chafetz N, Genant HK, Hoaglund FT (1982) Ischiogluteal tuberculous bursitis with progressive bony destruction. J Can Assoc Radiol 33: 119.

8 Ho G Jr., Tice AD, Kaplan SR (1978) Septic bursitis in the prepatellar and olecranon bursae; an analysis of 25 cases. Ann Intern Med 89: 21-27.

9 Canoso JJ, Checkman OT (1979) Septic subcutaneous bursitis: report of sixteen cases. J Rheumatol 6: 96-102.

10 Thompson GR, Manshady BM, Weiss JJ (1978) Septic bursitis. JAMA 240: 2280-2281.

11 Thornhill-Joynes M, Gonzales F, Stewart CA, Kanel GC, Lee GC, Capen DA et al (1986) Osteomyelitis associated with pressure ulcers. Arch Phys Med 67: 314-318.

12 Wanebo HJ, Webb E, Combs R (1965) Diagnosis of acute abdominal disease in paraplegic patients. California Med 103: 193-197.

13 Malik GM, Sapico FL, Montgomerie JZ (1982) Severe osteomyelitis in spinal cord injury patients. Arch Intern Med 142: 807-808. 